\title{
ESTUDO DA CINÉTICA DE SECAGEM, CONTRAÇÃO VOLUMÉTRICA E ANÁLISE DA DIFUSÃO LÍQUIDA DO MORANGO (FRAGARIA SP.)
}

\author{
Felipe Almeida de Sousa ${ }^{1}$ \\ Ednilton Tavares de Andrade ${ }^{2}$ \\ Vitor Gonçalves Figueira ${ }^{3}$ \\ Elisabeth de Moraes D'Andrea ${ }^{3}$ \\ Luiz Carlos Corrêa Filho ${ }^{3}$
}

\begin{abstract}
Resumo:O presente trabalho teve como objetivo o estudo da cinética de secagem, análise da contração volumétrica, bem como, obter os valores de difusão líquida durante o processo de secagem dos frutos do morango. Os frutos do morango foram submetidos à secagem nas temperaturas do ar de 40,55 e $70{ }^{\circ} \mathrm{C}$. Ao longo da secagem foi estudado o comportamento do fruto, como a variação do volume e perda de água, desde o início até os frutos chegaram a um teor de água de, aproximadamente, 0,25b.s. Aos dados experimentais foram aplicados modelos matemáticos capazes de descrever o processo de secagem e redução do volume. Os modelos de Midilli e Bala foram os que mais se adaptaram na descrição da cinética de secagem e contração volumétrica, respectivamente. A difusão líquida aumenta com a elevação da temperatura apresentando valores entre $1,14 \times 10^{-11}$ e $5,64 \times 10^{-11} \mathrm{~m}^{2} \mathrm{~s}^{-1}$.
\end{abstract}

Palavras-chave: morango; secagem; modelos matemáticos, difusão líquida.

\begin{abstract}
The present work aimed to study the drying kinetics, analysis of shrinkage, as well as obtaining the values of liquid diffusion during the process of drying the fruits of strawberry. The fruits of the strawberry were dried at temperatures of 40,55 and $70{ }^{\circ} \mathrm{C}$. During the drying behavior of the fruit has been studied as the change in volume and loss of water from the start until the fruits have reached a moisture content of approximately $0.25 \mathrm{db}$. Experimental data to mathematical models able to describe the drying process and reducing the volume were applied. The models Midilli and Bala were most adapted to describe the kinetics of drying and shrinkage, respectively. The liquid diffusion increases with increasing temperature with values between $1.14 \times 10^{-11}$ and $5.64 \times 10^{-11} \mathrm{~m}^{2} \mathrm{~s}^{-1}$.
\end{abstract}

Keywords: Strawberry; drying; mathematical models, liquid diffusion.

\footnotetext{
${ }^{1}$ PGEB, Universidade Federal Fluminense, felipealmeida_s89@hotmail.com.

2 DEG/UFLA, PGEB/Universidade Federal Fluminense, edniltontavares@gmail.com.

${ }^{3}$ PGEB, Universidade Federal Fluminense .
} 


\section{INTRODUÇÃO}

O morango (Fragaria sp.) é uma fruta com alto valor nutritivo, onde é possível encontrar compostos fenólicos, como a vitamina $\mathrm{C}$ e flavonoides, especialmente da classe das antocianinas. Estas substâncias presentes no morango contêm funções que permitem uma alta capacidade antioxidante, anticarcinogênica e antitrombótica, responsáveis por promover mais saúde aos consumidores do fruto (Mudnic et al., 2009).

A produção da cultura do morango na última década teve um crescimento bastante significativo para os pequenos produtores rurais dos estados Rio Grandes do Sul, Paraná, Minas Gerais, Espírito Santo e São Paulo. A rentabilidade da cultura do morango pode chegar a $224 \%$, sendo um fator favorável que pode ser associado ao crescimento de pequenos produtores agrícolas (Escobar, 2012).

Atualmente a produção brasileira média dos frutos do morango tem a sua maior parte destinada ao mercado in natura. A produtividade média por estado, em toneladas por hectare, é de 32,7 , no Rio Grande do Sul; 21,3, no Paraná; 25,2, em Minas Gerais; 34, no Espírito Santo; e 34, em São Paulo. O Brasil não consegue suprir toda a sua demanda de consumo, portanto importa da Argentina, Chile e Uruguai, países com alto potencial de exportação de frutos de morango (Escobar, 2012).

Os frutos de morango podem ser consumidos na forma natural ou semiprocessada. Para a conservação dos frutos são utilizados produtos naturais $\mathrm{e}$ biodegradáveis, que tem a função de evitar as modificações no sabor, cor e aroma do produto. A qualidade do produto é mantida com o uso de técnicas de resfriamento que são capazes de estender a vida útil do produto, mantendo suas características originais.

A conservação do morango normalmente é realizada com temperaturas baixas, que permite o armazenamento, transporte a distâncias longas e comercialização do produto, com o objetivo de reduzir perdas por deterioração e esmagamento. No processamento do fruto devem ser tomados cuidados essenciais, pois os mesmos apresentam uma alta perecibilidade na pós-colheita (Chitarra\& Chitarra, 2005).

Os prejuízos e perdas devido ao alto volume de produção de frutas tropicais podem ser controlados pela produção de passas. A faixa ideal de teor de água para estudos de secagem e armazenamento deve estar entre 0 - $25 \%$ de água adsorvida no produto (Hunt\&Pixton, 1974).

A secagem é o movimento da água do interior do produto até a superfície da face externa do fruto, esse processo envolve diferentes mecanismos de transferência de calor e massa. A secagem de produtos agrícolas permite um prolongamento na qualidade do produto e a colaboração na redução de perdas, promovendo um armazenamento do produto por um tempo mais longo, evitando, assim, a deterioração do produto.

Segundo Corrêa et al. (2001), o principal mecanismo de deslocamento da água no interior do produto agrícola, durante a secagem, é a difusão líquida, e com o caminhamento da secagem irá ocorrer o encolhimento e endurecimento da casca do produtos, podendo este processo de redução da água no produto ser representado pelas isotermas de sorção.

Para este estudo a umidade de equilíbrio, em base seca, dos frutos de morando, foi determinada, a partir do modelo de GAB (Guggenheim Anderson De Boer), descrito pela equação 1 a seguir (Anderson, 1946; Guggenheim, 1966; VandeBoer, 1953; Vanden Borg,1984):

$$
U_{e}^{*}=\frac{\mathrm{X}_{\mathrm{m}} \cdot \mathrm{C} \cdot \mathrm{K} \cdot \mathrm{A}_{\mathrm{a}}}{\left(1-\mathrm{K} \cdot \mathrm{A}_{\mathrm{a}}\right)\left(1-\mathrm{K} \cdot \mathrm{A}_{\mathrm{a}}+\mathrm{C} \cdot \mathrm{K} \cdot \mathrm{A}_{\mathrm{a}}\right)}
$$

em que, $U_{e}^{*}$ é o teor de água de equilíbrio, decimal (b.s.); $A_{a}$ é a atividade de água, em decimal; $\mathrm{X}_{\mathrm{m}}$ é o conteúdo de umidade na 
monocamada molecular, adimensional; $\mathrm{K}$ é o fator relacionado as multicamadas, adimensional; C é a constante de Guggenheim, adimensional.

As equações 2 e 3 são aplicadas na equação 1, para o cálculo do teor de água de equilíbrio em base seca.

$$
\begin{array}{r}
\mathrm{C}=\mathrm{C}_{\mathrm{o}} \exp \left(\frac{\Delta \mathrm{H}_{\mathrm{C}}}{\mathrm{R}_{\mathrm{gT}}}\right) \\
\mathrm{K}=\mathrm{K}_{\mathrm{o}} \exp \left(\frac{\Delta \mathrm{H}_{\mathrm{K}}}{\mathrm{R}_{\mathrm{gT}}}\right)
\end{array}
$$

em que, $R_{\mathrm{g}}$ é a constante universal do gás, em $\mathrm{J} \cdot \mathrm{mol}^{-1} \cdot \mathrm{K}^{-1} ; \quad \Delta \mathrm{H}_{\mathrm{C}} \quad$ é a variante de Guggenheim, em J.mol ${ }^{-1} \cdot \mathrm{K}^{-1} ; \Delta \mathrm{H}_{\mathrm{k}}$ é a variante de multicamadas, em J. $\mathrm{mol}^{-1} \cdot \mathrm{K}^{-1} ; \mathrm{C}_{\mathrm{o}}$ é a constante de $\mathrm{GAB}$, adimensional; $\mathrm{K}_{\mathrm{o}}$ é a constante de $\mathrm{GAB}$, adimensional; $\mathrm{T}$ é a temperatura, em ${ }^{\circ} \mathrm{C}$

A constante universal do gás $\mathrm{R}_{\mathrm{g}}=8,3143 \mathrm{~J} \cdot \mathrm{mol}^{-1} \cdot \mathrm{K}^{-1}$. Segundo Khalloufi et al. (2000) as constantes de GAB para o fruto do morango, assumem os seguintes valores $\Delta \mathrm{H}_{\mathrm{C}}=566,2 \mathrm{~J} \cdot \mathrm{mol}^{-1} \cdot \mathrm{K}^{-1} ; \Delta \mathrm{H}_{\mathrm{k}}=$ $-26,49 \mathrm{~J} \cdot \mathrm{mol}^{-1} \cdot \mathrm{K}^{-1} ; \mathrm{C}_{\mathrm{o}}=0,384 ; \mathrm{K}_{\mathrm{o}}=$ 1,099 .

A partir dos dados obtidos durante a secagem foram construídas as curvas de secagem em função do tempo. A razão de umidade e os dados de secagem foram expressos graficamente em função do tempo de secagem.

Conforme Brookeret al. (1992), o modelo de difusão líquida pode ser descrito segundo a lei de Fick que tem como objetivo estudar o comportamento da difusão da água em função do gradiente de concentração.

$$
\frac{d U^{*}}{d t}=\frac{d}{d x}\left(D \frac{d U^{*}}{d x}\right)
$$

em que $U^{*}$ é o teor de água do produto, decimal (b.s); D é o coeficiente de difusão líquida, $m^{2} s^{-1}$, T é o tempo, s e x é a distância em relação a um ponto de referência no corpo, $\mathrm{m}$.

Para os estudos de difusão líquida em materiais homogêneos pode-se utilizar a seguinte equação (Brookeret al., 1992):

$$
\frac{d U^{*}}{d t}=D\left[\frac{d^{2} U^{*}}{d r^{2}}+\frac{c}{r} \frac{d U^{*}}{d r}\right]
$$

em que, $\mathrm{r}$ é a espessura, $\mathrm{m} ; \mathrm{c}=0$ para corpos planos, 1 para corpos cilíndricos e 2 para corpos esféricos.

A razão de umidade do produto foi determinada por meio da equação descrita em Brookeret al. (1992), utilizada para forma geométrica tipo esférica.

$$
R U=\frac{U^{*}-U_{\mathrm{e}}^{*}}{U_{i}^{*}-U_{\mathrm{e}}^{*}}=\frac{6}{\pi^{2}} \sum_{\mathrm{n}=1}^{\infty} \frac{1}{\mathrm{n}^{2}} \exp \left[-\frac{\mathrm{n}^{2} \cdot \pi^{2} \cdot \mathrm{D} \cdot \mathrm{t}}{9} \cdot\left(\frac{3}{\mathrm{R}}\right)^{2}\right](6)
$$

em que, RU é a razão de umidade, adimensional; $\mathrm{R}$ é o raio equivalente, $\mathrm{m}$; $U_{e}^{*}$ é o teor de água de equilíbrio do produto, decimal (b.s.);n é o número de termos; $U_{i}^{*}$ é o teor de água inicial do produto, decimal (b.s.).

Para a determinação do coeficiente de difusão líquida os dados experimentais foram ajustados por meio de regressão não linear, no programa STATISTICA versão 5.0, pelo método Quasi-Newton.

Para a determinação das curvas de cinética de secagem foram utilizados os seguintes modelos matemáticos conforme mostra a tabela 1, e para a determinação das curvas de contração volumétrica foram utilizados os modelos matemáticos, apresentados na tabela 2. Os dados foram ajustados aos dados experimentais por meio de regressão não linear, no programa STATISTICA versão 5.0, pelo método Quasi-Newton.

Segundo Corrêa et al. (2001) a taxa de redução de água é definida como a quantidade de água que um determinado produto perde por unidade de matéria seca do produto por unidade de tempo, conforme mostra a equação 26.

$$
\mathrm{TRA}=\frac{\mathrm{Ma}_{\mathrm{o}}-\mathrm{Ma}_{\mathrm{i}}}{\mathrm{M}_{\mathrm{s}}\left(\mathrm{t}_{\mathrm{i}}-\mathrm{t}_{\mathrm{o}}\right)}
$$

em que, TRA é a taxa de redução de água, em $\mathrm{kg} . \mathrm{kg}^{-1} \cdot \mathrm{h}^{-1} ; \mathrm{M}_{\mathrm{ao}}$ é a massa de água total anterior, em $\mathrm{kg} ; \mathrm{M}_{\mathrm{ai}}$ é a massa de água total atual, em kg; Ms é a matéria seca, em kg; $\mathrm{t}_{\mathrm{o}}$ é o tempo total de secagem anterior, em $h$; $t_{i}$ é o tempo total de secagem atual, em $\mathrm{h}$. 
Tabela 1.Modelos matemáticos utilizados para predizer o fenômeno de cinética de secagem de produtos agrícolas.

\begin{tabular}{|c|c|}
\hline Model & Equação \\
\hline (7) & $\mathrm{RU}=\exp \left(-k \mathrm{t}^{\mathrm{n}}\right)$ \\
\hline (8) & $R U=a \exp (-k t)+(1-a) \exp (-k b t)$ \\
\hline (9) & $R U=a \exp \left(-k_{0} t\right)+b \exp \left(-k_{1} t\right)$ \\
\hline$(10)$ & $R U=a \exp (-k t)+(1-a) \exp (-k a t)$ \\
\hline$(11)$ & $\mathrm{RU}=\operatorname{aexp}(-\mathrm{kt})+\mathrm{c}$ \\
\hline$(12)$ & $R U=a \exp (-k t)+b \exp \left(-k_{0} t\right)+c \exp \left(-k_{1} t\right)$ \\
\hline (13) & $\mathrm{RU}=\operatorname{aexp}(-\mathrm{kt})+\mathrm{c}$ \\
\hline (14) & $\mathrm{RU}=\operatorname{aexp}\left(-k t^{\mathrm{n}}\right)+\mathrm{bt}$ \\
\hline$(15)$ & $\mathrm{RU}=\exp \left(\left(-\mathrm{a}\left(\mathrm{a}^{2}+4 b t\right)^{0,5}\right) / 2 b\right)$ \\
\hline$(16)$ & $R U=\operatorname{aexp}(-k t)+(1-a) \exp (-k t)$ \\
\hline
\end{tabular}

em que, (7) Modelo de Page; (8) Aproximação da Difusão; (9) Dois Termos; (10) Exponencial de Dois Termos; (11) Henderson e Pabis; (12) Henderson e Pabis Modificado; (13) Logarítmico; (14) Midilli; (15) Thompson; (16) Verna. RU é a razão de umidade do produto, adimensional; $t$ é o tempo de secagem, em $\mathrm{h} ; \mathrm{k}, \mathrm{k}_{0}, \mathrm{k}_{1}$ são coeficientes de secagem, em $h^{-1} ; \mathrm{a}, \mathrm{b}, \mathrm{c}, \mathrm{n}$ são constantes dos modelos, adimensional; Brookeret al. (1992), Corrêa et al. (2001), Alves et al. (2013).

Tabela 2.Modelos matemáticos utilizados para predizer o fenômeno de contração volumétrica de produtos agrícola.

\begin{tabular}{|c|c|}
\hline $\begin{array}{c}\text { Mode } \\
\text { o }\end{array}$ & Equação \\
\hline$(17)$ & $\Psi=\left(\mathrm{a}\left(\exp \left(\mathrm{b} U^{*}\left(\mathrm{~T}^{\mathrm{c}}\right)\right)\right)\right)$ \\
\hline (18) & $\Psi=\left(\mathrm{a}+\left(\mathrm{b} U^{*}\right)\right) \mathrm{T}^{\mathrm{c}}$ \\
\hline (19) & $\Psi=\left(1+\left(\mathrm{a}\left(U^{*}-\mathrm{U}_{0}\right)\right)\right) \mathrm{T}^{\mathrm{b}}$ \\
\hline (20) & $\Psi=\left(1-\left(\mathrm{a}\left(1-\exp \left(-\mathrm{b}\left(\mathrm{U}_{0}-U^{*}\right)\right)\right)\right)\right) \mathrm{T}^{\mathrm{c}}$ \\
\hline (21) & $\Psi=1 /\left(\mathrm{a}+\mathrm{b} \exp \left(U^{*}\right)\right) \mathrm{T}^{\mathrm{c}}$ \\
\hline$(22)$ & $\Psi=1-\mathrm{a}^{*}\left(1-\exp \left(-\mathrm{b} *\left(\left(\mathrm{U}_{0} / 100\right)-\left(U^{*} / 100\right)\right)\right)\right)$ \\
\hline$(23)$ & $\Psi=\left(\mathrm{a}+\mathrm{b} U^{*}+\mathrm{C} U^{* 2}\right) \mathrm{T}^{\mathrm{c}}$ \\
\hline (24) & $\Psi=\mathrm{a}^{*} \operatorname{Exp}\left(\mathrm{b}^{*}\left(U^{*} / 100\right)\right)$ \\
\hline (25) y & $\Psi=\mathrm{a}+\mathrm{b}^{*}\left(U^{*} / 100\right)$ \\
\hline $\begin{array}{l}\text { em que, } \\
\text { Adaptad } \\
\text { Woods } \\
\text { Polinomi } \\
\text { índice de } \\
\text { teor de á } \\
\text { de água } \\
\text { do ar de }\end{array}$ & $\begin{array}{l}\text { e,(17) Exponencial Adaptado; (18) Linear } \\
\text { do; (19) Rahman Adaptado; (20) Bala \& } \\
\text { Adaptado; (21) Correa; (22) Bala; (23) } \\
\text { nial; (24) Exponencial; (25) Linear. } \Psi \text { é o } \\
\text { de contração volumétrica, em decimal; } U^{*} \text { é o } \\
\text { água do produto, decimal (b.s); } \mathrm{U}_{0} \text { é o teor } \\
\text { inicial, em \% base seca; T é a temperatura } \\
\text { e secagem, em }{ }^{\circ} \mathrm{C} ; \text { a, b, c são constantes dos } \\
\text { s, adimensional. }\end{array}$ \\
\hline
\end{tabular}

Para análise de representatividade do modelo de secagem, os dados experimentais foram comparados com os valores estimados por cada modelo, verificando-se a porcentagem de erro médio relativo $(\mathrm{P})$ e o erro médio estimado (SE), de acordo, respectivamente, com as equações a seguir (Ryan, 2009).

$$
\begin{aligned}
& \mathrm{P}=\frac{100}{\mathrm{n}} \sum \frac{\left|\mathrm{Y}-\mathrm{Y}_{0}\right|}{\mathrm{Y}} \\
& \mathrm{SE}=\sqrt{\sum \frac{\left(\mathrm{Y}-\mathrm{Y}_{0}\right)^{2}}{\mathrm{GLR}}}
\end{aligned}
$$

$$
\chi^{2}=\sum \frac{\left(\mathrm{Y}-\mathrm{Y}_{0}\right)^{2}}{\mathrm{GLR}}(29)
$$

em que, $Y$ é o valor observado experimentalmente; $\mathrm{Y}_{0}$ é o valor calculado pelo modelo; $\mathrm{n}$ é o número de observações experimentais; GLR são os graus de liberdade do modelo.

\section{MATERIAS E MÉTODOS}

O presente trabalho foi desenvolvido no Laboratório de Termociência (LATERMO), da Universidade Federal Fluminense - UFF, Niterói, RJ. Foi realizado o estudo da cinética de secagem, difusão líquida e contração volumétrica dos frutos do morango para as temperaturas do ar de secagem de $40^{\circ} \mathrm{C}, 55^{\circ} \mathrm{C}$ e $70^{\circ} \mathrm{C}$.

Os frutos do morango foram cortados longitudinalmente em duas partes com auxílio de uma faca inox, com objetivo de obter um material com forma geométrica simétrica e homogênea.

Utilizou-se um secador de bandejas com controle de temperatura e vazão de ar. $\mathrm{O}$ secador estacionário agregou três amostras de morango em três bandejas perfuradas, para cada temperatura, que foram pesadas em vários intervalos de tempo. Foi observada a massa inicial, e realizadas pesagens nas amostras até que chegassem a um teor de água próximo a 0,25 b.s., sendo esses procedimentos adotados para cada temperatura estudada. 
$\mathrm{O}$ ar de secagem foi aquecido por um conjunto de lâmpadas na base do secador, sendo conduzido, de forma homogênea, até as bandejas, por meio de um ventilador com circulação forçada de ar, com velocidade de $1,0 \mathrm{~m} \cdot \mathrm{s}^{-1}$.

No decorrer de cada secagem foram realizadas medições de umidade relativa do ar e temperatura ambiente, por meio de um termo-higrômetro digital, sendo considerados, para os cálculos experimentais, os dados médios obtidos durante cada secagem. A partir dos dados de umidade relativa do ar ambiente e temperatura média do ar, foi possível à determinação da umidade relativa do ar de secagem com auxílio do software GRAPSI.

Durante a secagem foi determinado o quanto de massa de água era perdido ao longo de todo o intervalo de tempo, em paralelo foi estudada a redução das dimensões: comprimento, largura e espessura, com o auxílio de um paquímetro digital.

As medições das condições iniciais de teor de água da amostra foram determinadas com o uso do método da estufa descrito pelo Instituto Adolfo Lutz (1985), para cada temperatura do ar estudada. Com os dados obtidos experimentalmente foi possível obter as curvas de cinética de secagem, contração volumétrica e difusão líquida, com o ajuste de modelos matemáticos.

As análises da contração volumétrica e da difusão líquida do fruto do morango foram realizadas a partir da aproximação da forma do fruto do morango para um cone elíptico, o qual permitiu uma melhor representação do volume, sendo obtidas as suas dimensões por meio de um paquímetro digital ao longo de toda a secagem.

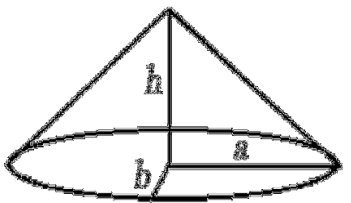

Figura 1. Desenho esquemático de um cone elíptico com suas dimensões características.

O volume determinado no modelo de contração volumétrica de um cone elíptico pode ser descrito pela equação abaixo (Mohseninet al., 1986).

$$
\mathrm{V}=\frac{\pi(\mathrm{ahb})}{3}
$$

$O$ índice de contração volumétrica do morango ( $\Psi)$, durante a secagem foi determinado pela relação entre o volume para cada teor de água e o volume inicial, de acordo com a equação:

$$
\Psi=\frac{\mathrm{V}}{\mathrm{V}_{0}}
$$

em que, $\Psi$ é o índice de contração volumétrica, em decimal; V é o volume em cada teor de água, em $\mathrm{m}^{3} ; \mathrm{V}_{0}$ é o volume inicial, $\mathrm{em}^{3}$.

$\mathrm{O}$ raio equivalente usado no modelo de difusão líquida é o da esfera equivalente, determinado pelo volume da esfera, a partir da medição dos três eixos ortogonais da esfera, utilizando a equação descrita emMohseninet al. (1986).

\section{RESULTADOS E DISCUSSÕES}

A Figura 2 apresenta as curvas de secagem dos frutos de morango com diferentes temperaturas do ar de secagem. Conforme apresentado na figura o aumento da temperatura do ar de secagem provoca uma redução no tempo de secagem, ou seja, são inversamente proporcionais.

A partir dos modelos de cinética de secagem estudados pode-se concluir que o de Midilli foi o que apresentou o menor valor de erro médio relativo (abaixo que $\mathrm{P}=2,27 \%$ ), de erro estimado (menor que $\mathrm{SE}=0,022$ )e de qui-quadrado (menor que $\left.\chi^{2}=0,0001\right)$ e maior coeficiente de determinação $\left(\mathrm{R}^{2}=99,98 \%\right)$. Exceto para o modelo de Thompson, todos os modelos 
estudados representaram satisfatoriamente a cinética de secagem dos frutos de morango em diferentes temperaturas do ar de secagem, com erro médio relativo abaixo de $10 \%$ e coeficiente de determinação acima de $99,61 \%$, podendo, assim, serem utilizados em outras aplicações.

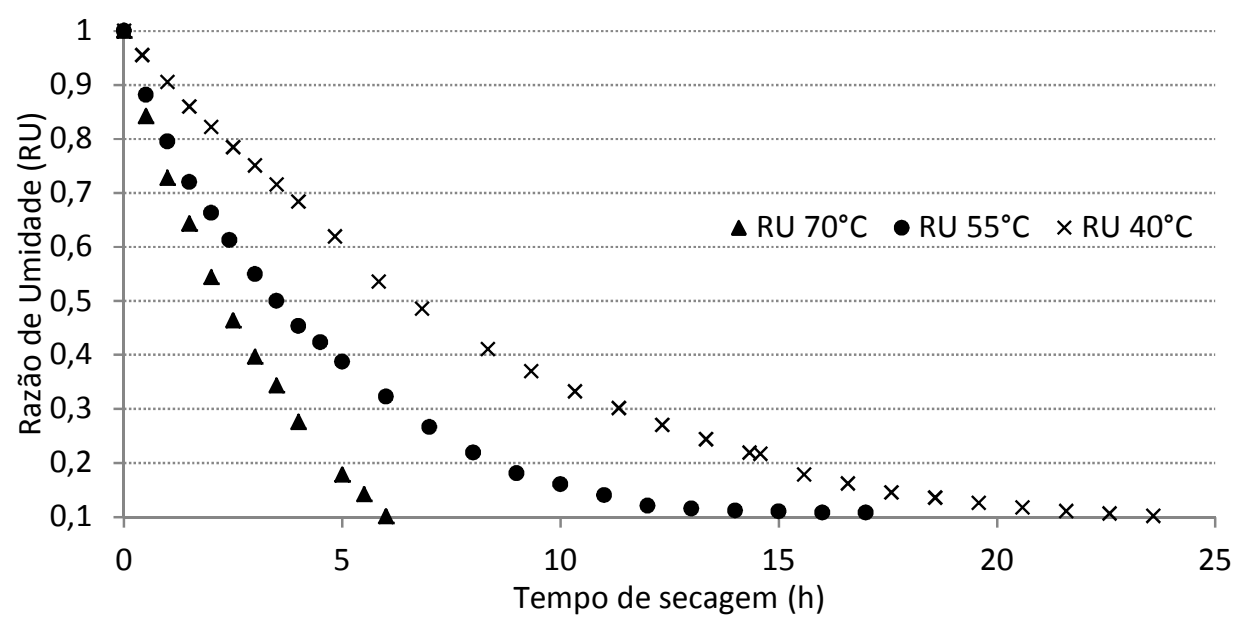

Figura 2. Variações da razão de umidade, na secagem do morango, em função do tempo para diferentes temperaturas do ar de secagem.

Os estudos das curvas de secagem dos frutos de morango foram realizados com objetivo de observar o comportamento da secagem durante o tempo, para cada temperatura estudada, conforme pode ser visto nas Figuras 3, 4 e 5 , que apresentam a razão de umidade experimental e simulada pelo modelo de Midilli, e a taxa de redução de água dos frutos de morango.

A taxa de redução de água durante a secagem dos frutos de morango se apresenta homogênea para todas as temperaturas estudadas, indicando que a secagem foi realizada de forma satisfatória e equilibrada.
Para os modelos estudados de contração volumétrica somente os modelo de Bala e o de Bala \& Woods Adaptado apresentaram erro médio relativo inferior a $10 \%$, mas o modelo de Bala apresentou um resultado mais satisfatório (menor $\mathrm{P}, \mathrm{SE} \mathrm{e}$ $\chi^{2}$ e maior $R^{2}$ ), sendo, assim, o utilizado para apresentação dos resultados.

As Figuras 6, 7 e 8 apresentam os valores de contração volumétrica experimental e simulado pelo modelo Bala em função do tempo de secagem para as temperaturas do ar de $40^{\circ} \mathrm{C}, 55^{\circ} \mathrm{C}$ e $70^{\circ} \mathrm{C}$.

A Figura 9 apresenta o índice de contração volumétrica simulado para as temperaturas estudadas. 
Tabela 3.Parâmetros obtidos nos modelos de cinética de secagem para as temperaturas de $40^{\circ} \mathrm{C}$, $55^{\circ} \mathrm{C}$ e $70^{\circ} \mathrm{C}$

\begin{tabular}{|c|c|c|c|c|c|c|c|c|c|c|c|}
\hline \multirow[b]{2}{*}{ Modelo } & \multicolumn{11}{|c|}{ Temperatura $40^{\circ} \mathrm{C}$} \\
\hline & $\mathbf{R}^{2}$ & $\mathbf{P}$ & SE & $\chi^{2}$ & $\mathbf{A}$ & $\mathbf{K}$ & B & Ko & k1 & $\mathrm{C}$ & $\mathbf{N}$ \\
\hline $\begin{array}{l}\text { Aprox. da } \\
\text { difusão }\end{array}$ & 99,94 & 3,72 & 0,045 & 0,0020 & 0,9999 & 0,1052 & $-2,037$ & - & - & - & - \\
\hline Dois termos & 99,95 & 3,11 & 0,032 & 0,0010 & 0,0002 & - & 1,0151 & $-0,19$ & 0,108 & - & - \\
\hline $\begin{array}{l}\text { Exp. de dois } \\
\text { termos }\end{array}$ & 99,85 & 8,37 & 0,099 & 0,0098 & 0,6295 & 0,1233 & - & - & - & - & - \\
\hline $\begin{array}{l}\text { Henderson e } \\
\text { Pabis }\end{array}$ & 99,84 & 9,38 & 0,102 & 0,0104 & 1,0064 & 0,104 & - & - & - & - & - \\
\hline $\begin{array}{c}\text { Henderson e } \\
\text { Pabis modificado }\end{array}$ & 99,95 & 3,11 & 0,025 & 0,0006 & 0,1656 & 0,1076 & 0,0002 & $-0,19$ & 0,108 & 0,85 & - \\
\hline Logarítmico & 99,90 & 5,58 & 0,056 & 0,0031 & 0,9851 & 0,1154 & - & - & - & 0,036 & - \\
\hline Midilli & 99,98 & 2,27 & 0,022 & 0,0005 & 0,9875 & 0,0826 & 0,0022 & - & - & - & 1,1346 \\
\hline Page & 99,83 & 9,38 & 0,103 & 0,0106 & - & 0,103 & - & - & - & - & 1,0007 \\
\hline Thompson & 92,52 & 46,22 & 0,675 & 0,4556 & 0,1035 & - & 0,0971 & - & - & - & - \\
\hline \multirow{2}{*}{ Verna } & 99,83 & 9,37 & 0,103 & 0,0106 & 0,1000 & 0,1032 & - & - & - & - & - \\
\hline & \multicolumn{11}{|c|}{ Temperatura $55^{\circ} \mathrm{C}$} \\
\hline Modelo & $\mathbf{R}^{2}$ & $\mathbf{P}$ & SE & $\chi^{2}$ & $\mathbf{A}$ & $\mathbf{K}$ & $\mathbf{B}$ & Ko & k1 & $\mathbf{C}$ & $\mathbf{N}$ \\
\hline $\begin{array}{l}\text { Aprox. da } \\
\text { difusão }\end{array}$ & 99,88 & 8,34 & 0,051 & 0,00260 & 0,4414 & 0,3205 & 1 & - & - & - & - \\
\hline Dois termos & 99,61 & 8,06 & 0,041 & 0,00168 & 0,5051 & - & 0,5051 & 0,324 & 0,324 & - & - \\
\hline $\begin{array}{l}\text { Exp. de dois } \\
\text { termos }\end{array}$ & 99,8 & 5,16 & 0,052 & 70 & 1,5599 & 0,3997 & - & - & - & - & - \\
\hline $\begin{array}{l}\text { Henderson e } \\
\text { Pabis }\end{array}$ & 99,72 & 8,06 & 0,071 & 0,00504 & 0,3245 & 0,3245 & - & - & - & - & - \\
\hline $\begin{array}{c}\text { Henderson e } \\
\text { Pabis modificado }\end{array}$ & 99,72 & 8,06 & 0,032 & 0,00102 & 0,3368 & 0,3245 & 0,3367 & 0,324 & 0,324 & 0,337 & - \\
\hline Logarítmico & 99,96 & 1,60 & 0,019 & 0,00036 & 1,1521 & 0,2404 & - & - & - & $-0,17$ & - \\
\hline Midilli & 99,98 & 1,19 & 0,010 & 0,00010 & 0,9985 & 0,2781 & $-0,025$ & - & - & - & 0,8973 \\
\hline Page & 99,82 & 5,65 & 0,057 & 0,00325 & - & 0,2919 & - & - & - & - & 1,0842 \\
\hline Thompson & 90,58 & 40,28 & 0,404 & 0,16322 & 0,2657 & - & 0,2606 & - & - & - & - \\
\hline \multirow[t]{2}{*}{ Verna } & 99,71 & 8,34 & 0,072 & 0,00518 & 0,1000 & 0,3205 & - & - & - & - & - \\
\hline & \multicolumn{11}{|c|}{ Temperatura $7^{\circ} \mathrm{C}$} \\
\hline Modelo & $\mathbf{R}^{2}$ & $\mathbf{P}$ & SE & $x^{2}$ & $\mathbf{A}$ & $\mathbf{K}$ & $\mathbf{B}$ & K0 & k1 & $\mathbf{C}$ & $\mathbf{N}$ \\
\hline $\begin{array}{l}\text { Aprox. da } \\
\text { difusão }\end{array}$ & 99,94 & 3,72 & 0,045 & 0,00203 & 999 & 0,1052 & $-2,037$ & - & - & - & - \\
\hline Dois termos & 99,95 & 3,11 & 0,032 & 0,00102 & 0,0002 & - & 1,0151 & $-0,19$ & 0,108 & - & - \\
\hline $\begin{array}{l}\text { Exp. de dois } \\
\text { termos }\end{array}$ & 99,85 & 8,37 & 0,099 & 0,00980 & 0,6295 & 0,1233 & - & - & - & - & - \\
\hline $\begin{array}{l}\text { Henderson e } \\
\text { Pabis }\end{array}$ & 99,84 & 9,38 & 0,102 & 0,01040 & 1,0064 & 0,104 & - & - & - & - & - \\
\hline $\begin{array}{c}\text { Henderson e } \\
\text { Pabis modificado }\end{array}$ & 99,95 & 3,11 & 0,025 & 0,00063 & 0,1656 & 0,1076 & 0,0002 & $-0,19$ & 0,108 & 0,85 & - \\
\hline Logarítmico & 99,90 & 5,58 & 0,056 & 0,00314 & 0,9851 & 0,1154 & - & - & - & 0,036 & - \\
\hline Midilli & 99,98 & 2,27 & 0,022 & 0,00048 & 0,9875 & 0,0826 & 0,0022 & - & - & - & 1,1346 \\
\hline Page & 99,83 & 9,38 & 0,103 & 0,01061 & - & 0,103 & - & - & - & - & 1,0007 \\
\hline Thompson & 92,52 & 46,22 & 0,675 & 0,45563 & 0,1035 & - & 0,0971 & - & - & - & - \\
\hline Verna & 99,83 & 9,37 & 0,103 & 0,01061 & 0,1000 & 0,1032 & - & - & - & - & - \\
\hline
\end{tabular}




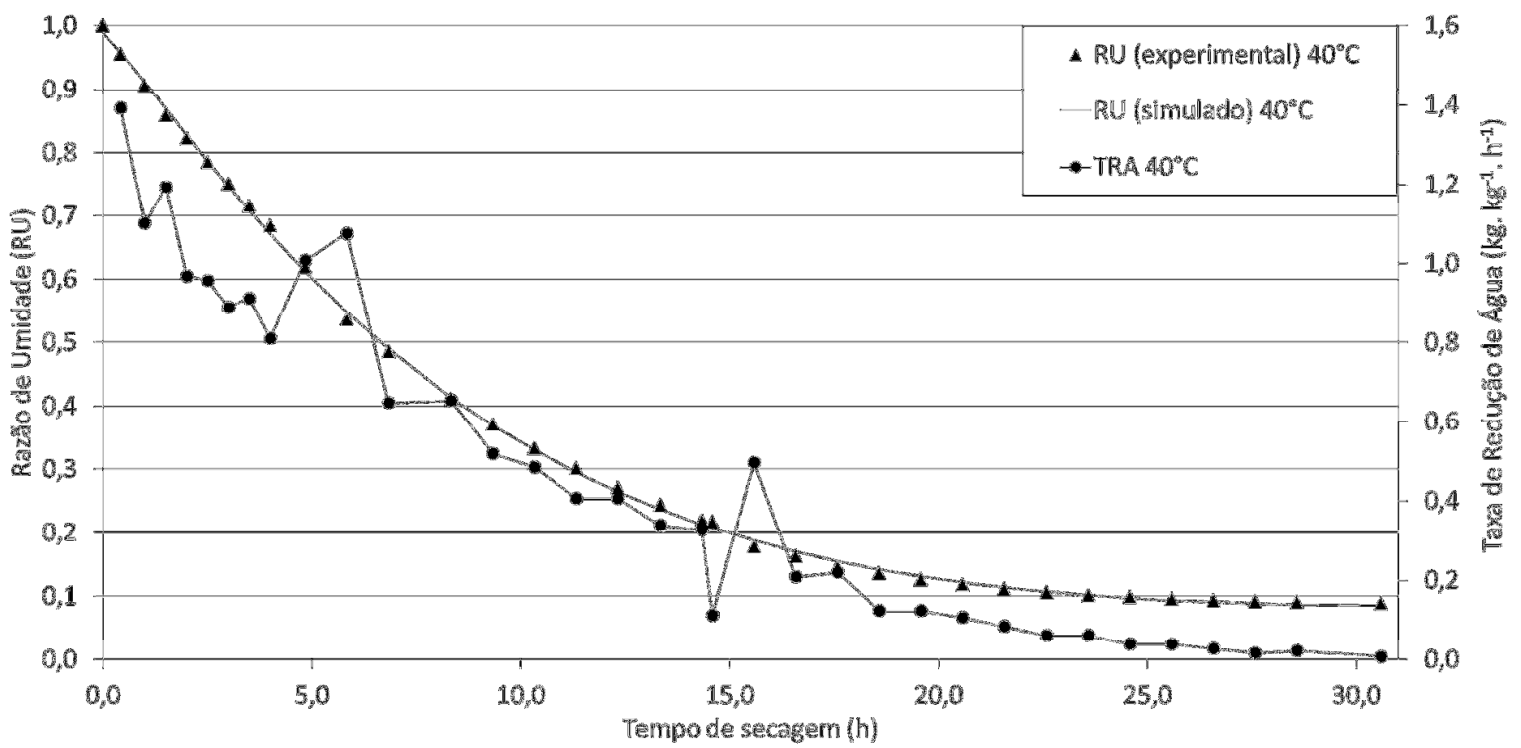

Figura 3. Curva de razão de umidade, experimental e simulada pelo modelo de Midilli para temperatura do ar de $40^{\circ} \mathrm{C}$.

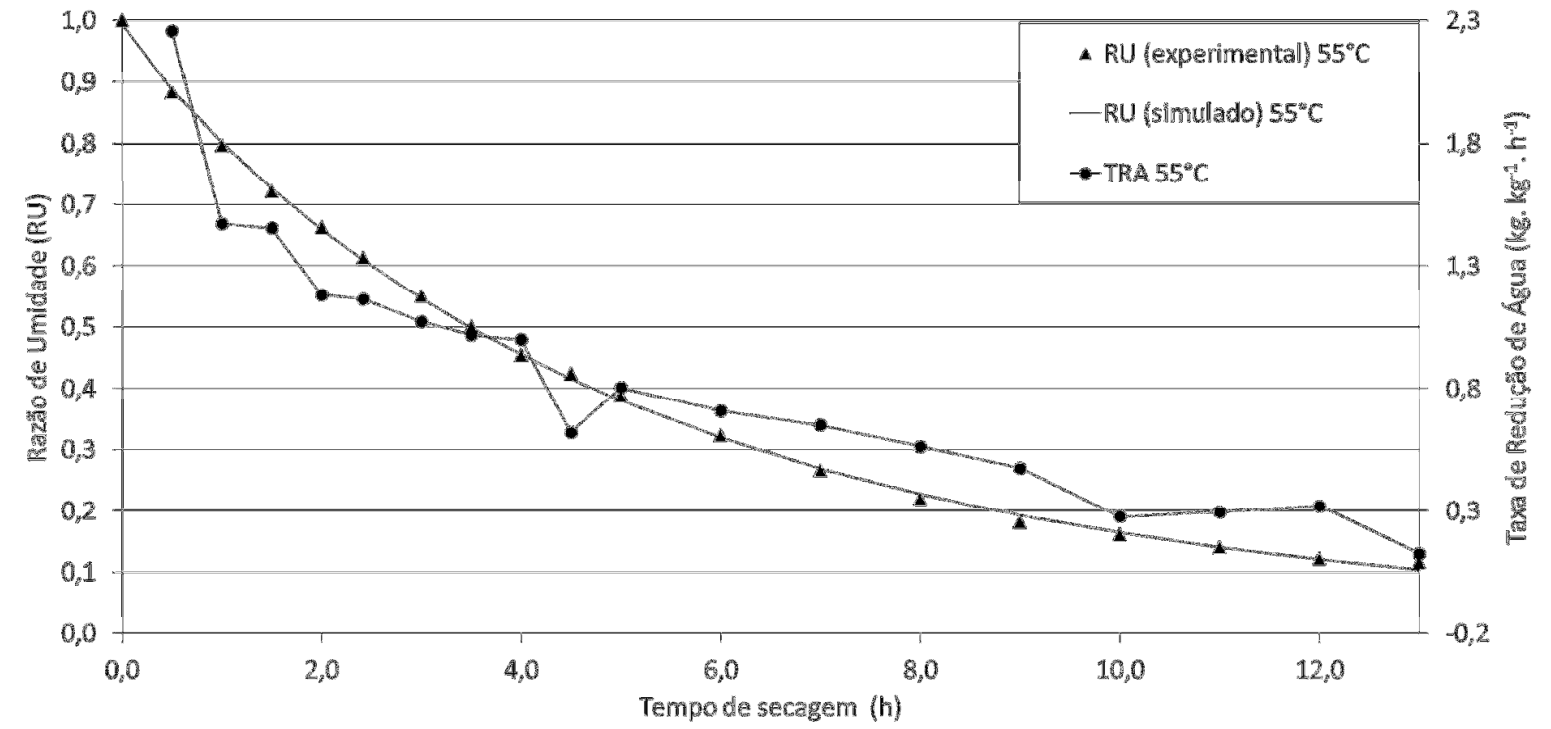

Figura 4. Curva de razão de umidade, experimental e simulada pelo modelo de Midilli para temperatura do ar de $55^{\circ} \mathrm{C}$. 


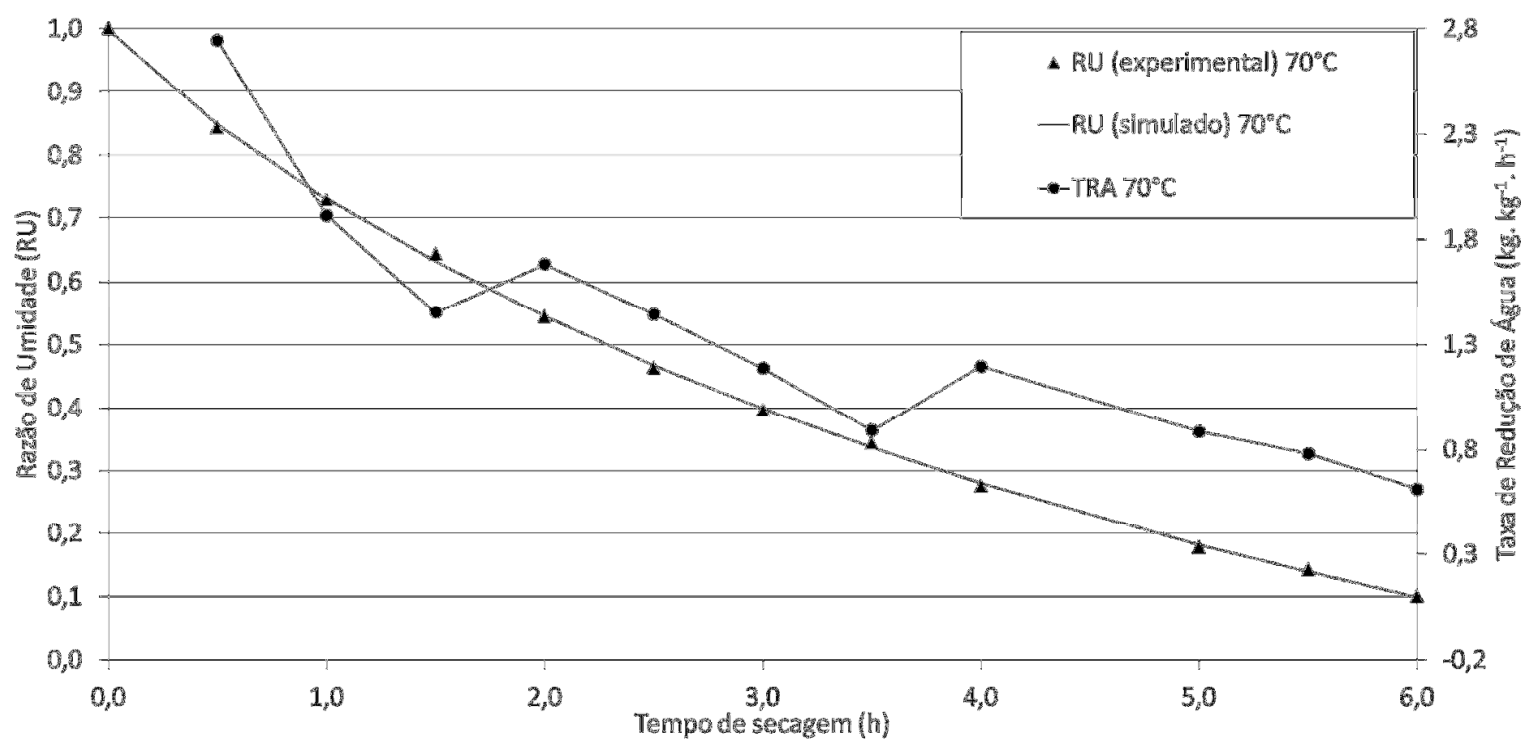

Figura 5. Curva de razão de umidade, experimental e simulada pelo modelo de Midilli para temperatura do ar de $70^{\circ} \mathrm{C}$.

Tabela 4. Parâmetros obtidos nos modelos de contração volumétrica para as temperaturas do ar de $40^{\circ} \mathrm{C}, 55^{\circ} \mathrm{C}$ e $70^{\circ} \mathrm{C}$.

\begin{tabular}{lccccccc}
\hline \multicolumn{1}{c}{ Modelo } & $\mathbf{a}$ & $\mathbf{b}$ & $\mathbf{c}$ & $\mathbf{P}(\boldsymbol{\%})$ & $\mathbf{S E}(\mathbf{d e c})$ & $\chi^{2}$ & $\mathbf{R}^{\mathbf{2}}$ \\
\hline Exponencial Adaptado & 0,22924 & 0,10197 & 0,03405 & 15,2316 & 0,3881 & 0,1506 & 93,58 \\
Linear Adaptado & 0,02768 & 0,04776 & 0,1081 & 13,1494 & 0,1930 & 0,0372 & 98,43 \\
Rahman Adaptado & 0,07914 & $-0,0106$ & - & 12,4461 & 0,2584 & 0,0668 & 97,21 \\
Bala \& Woods Adaptado & 1,33383 & 0,08551 & 0,00352 & 9,0164 & 0,1374 & 0,0189 & 98,15 \\
Corrêa & 17,9703 & $-14,94$ & - & 22,5576 & 0,3482 & 0,1212 & 89,43 \\
Bala & 1,40363 & 7,85171 & - & 8,9267 & 0,0877 & 0,0077 & 98,40 \\
Polinomial & 0,06775 & 6,56723 & 4,65565 & 12,5252 & 0,3414 & 0,1166 & 95,07 \\
Exponencial & 0,22898 & 11,6751 & - & 15,3761 & 0,2247 & 0,0505 & 93,54 \\
Linear & 0,04667 & 7,26908 & - & 13,0546 & 0,3421 & 0,1170 & 95,05 \\
\hline
\end{tabular}

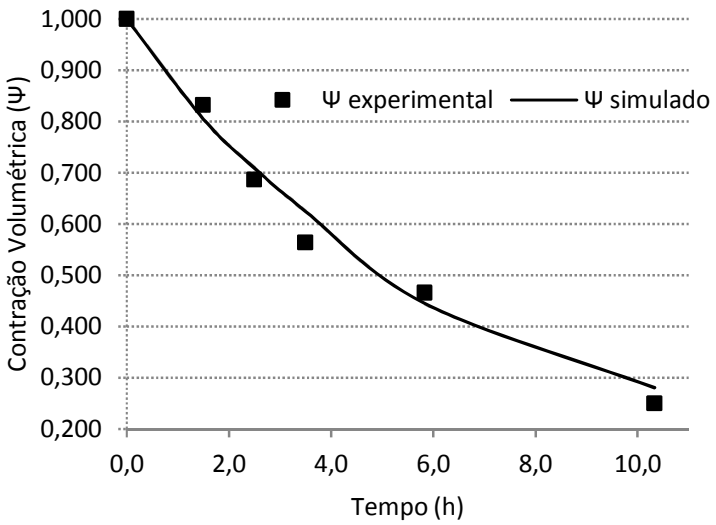

Figura 6. Índice de contração volumétrica dos frutos de morango durante a secagem em temperatura do ar de $40^{\circ} \mathrm{C}$.

ENGEVISTA, V. 16, n. 1, p.8-18 Março 2014

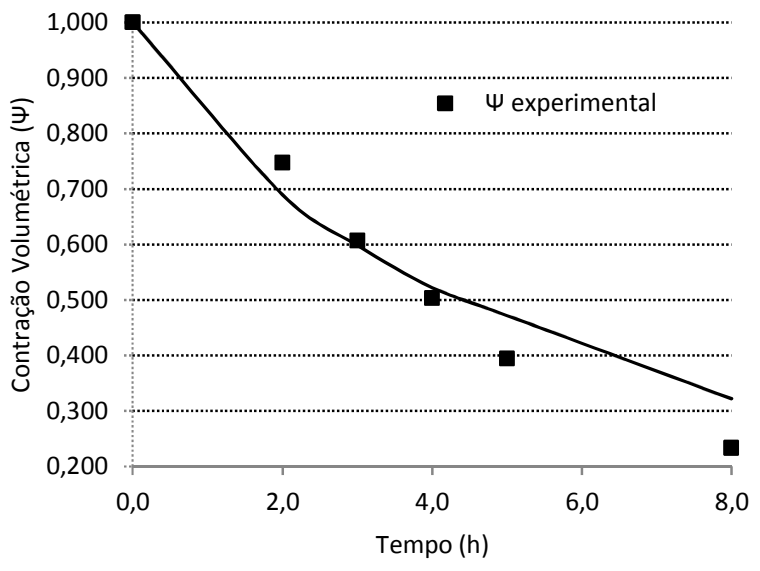

Figura 7. Índice de contração volumétrica dos frutos de morango durante a secagem em temperatura do ar de $55^{\circ} \mathrm{C}$. 


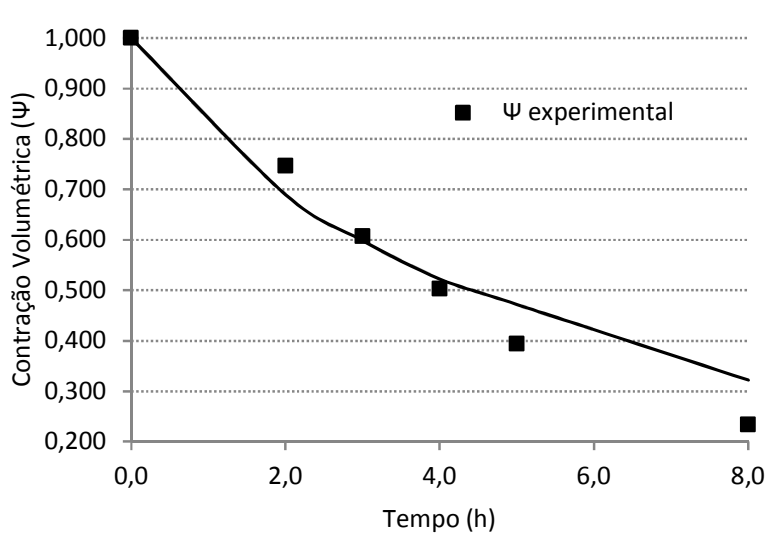

Figura 8. Índice de contração volumétrica dos frutos de morango durante a secagem em temperatura do ar de $70^{\circ} \mathrm{C}$.

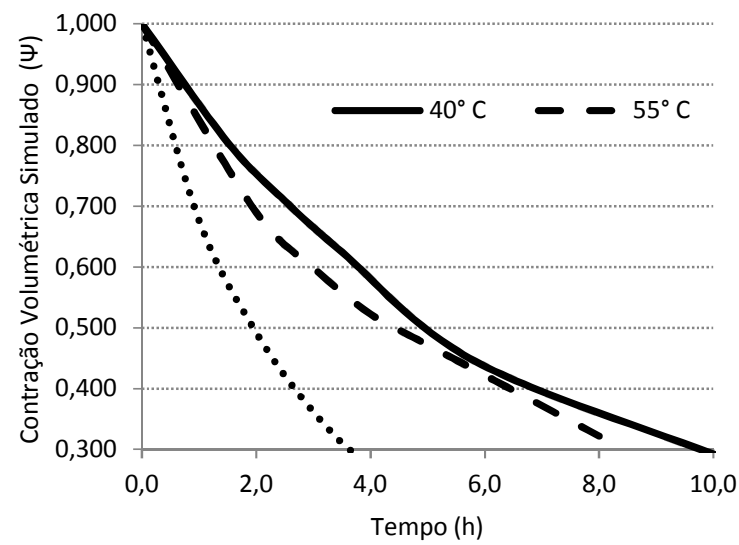

Figura 9. Índice de contração volumétrica simulado dos frutos de morango durante a secagem em temperaturas do ar de 40, 55 e $70^{\circ} \mathrm{C}$.

Os valores do índice de contração volumétrica determinados experimentalmente estiveram próximos do simulado conforme mostram as Figuras 6, 7 e 8 .

A contração volumétrica ocorreu de forma mais acelerada no início da secagem para todas as temperaturas, sendo que para a temperatura do ar de $70^{\circ} \mathrm{C}$ houve uma redução do volume maior em um intervalo de tempo menor em relação às outras temperaturas estudas, conforme observado.

Os resultados obtidos de difusão líquida para as temperaturas do ar de secagem de de $40^{\circ} \mathrm{C}, 55^{\circ} \mathrm{C}$ e $70^{\circ} \mathrm{C}$, foram:

ENGEVISTA, V. 16, n. 1, p.8-18 Março 2014
$1,14 \times 10^{-11}, 2,61 \times 10^{-11} \mathrm{e} 5,64 \times 10^{-11} \mathrm{~m}^{2} \mathrm{~s}^{-1}$, respectivamente.

Pelos resultados obtidos experimentalmente foi possível perceber que os valores do coeficiente de difusão líquida do morango aumentam em função da temperatura.

Segundo Madambaet al. (1996), os valores para difusão líquida de produtos agrícolas estão na ordem de grandeza de $10^{-9}$ a $10^{-11} \mathrm{~m}^{2} \mathrm{~s}^{-1}$, sendo que resultados obtidos experimentalmente de difusão liquida dos frutos de morango se encontram nessa ordem de grandeza.

\section{CONCLUSÃO}

Para o estudo do comportamento da secagem e da contração volumétrica dos frutos do morango, nas temperaturas do ar de 40,55 e $70^{\circ} \mathrm{C}$, pode-se concluir que:

A temperatura do ar de secagem de $70^{\circ} \mathrm{C}$ propiciou o menor tempo de secagem e maior rapidez na contração volumétrica dos frutos dentre as temperaturas estudas.

O modelo de Midilli dentre os modelos de simulação da cinética de secagem foi o que apresentou melhor resultado.

O modelo de Bala, dentre os modelos de simulação da contração volumétrica foi o que apresentou melhor ajuste.

A difusão líquida aumenta com a elevação da temperatura apresentando valores entre $1,14 \times 10^{-11} \mathrm{e} 5,64 \times 10^{-}$ ${ }^{11} \mathrm{~m}^{2} \mathrm{~s}^{-1}$.

\section{AGRADECIMENTOS}

À CAPES pelo apoio financeiro.

\section{BIBLIOGRAFIA}

ALVES, G. E.; ISQUIERDO, E. P.; BORÉM, F. M.; SIQUEIRA, V. C., OLIVEIRA, P. D.; ANDRADE, E. T. 
Cinética de secagem de café natural para diferentes temperaturas e baixa umidade relativa. Coffee Science, Lavras, v. 8, n. 2, p. 238-247, 2013.

ANDERSON, R. B.; Modification of BET equation.Journal of American Chemical Society, 68, 686-691, 1946.

BROOKER, D.B.; BAKKER-ARKEMA, F.W.; HALL, C.W. Drying and storage of grains and oilseeds.Westport: The AVI PublishingCompany, 1992. 450 p.

CHITARRA, M., \& CHITARRA, A. Póscolheita de frutas e hortaliças: Fisiologia e manuseio. ESAL FAEPE, 78p. 2005.

CORRÊA, P. C.; MACHADO, P. F.; ANDRADE, E. T. Cinética de secagem e qualidade de grãos de milho-pipoca. Ciência e Agrotecnologia, Lavras, v. 25, p. 134-142, 2001.

CHEN, C.; JAYAS, D. S. Evaluation of the GAB equation for the isotherms of agricultural products. Transactions of ASAE, St. Joseph, v. 41, n. 6, p. 1755-1760, 1998.

ESCOBAR, J.O Morango é a nova árvore do conhecimento da Agência Embrapa de Informação. Jornal O Trombeta, Capanema, Ed 1088, p. 1-32, 2012.

GUGGENHEIM, E. A. Application of statistical mechanics.[S.1.]: Clarendon Press. 1966.

HUNT, W. H.; PIXTON, S. W. Moisture its significance, behavior, and measurement. In: CHRISTENSEN, C. M. (Ed.). Storage of cereal grains and their products. 2. ed. St. Paul: American Association of Cereal Chemists, 1974. p. 1-55.
INSTITUTO ADOLFO LUTZ. Normas Analíticas do Instituto Adolfo Lutz. [S.1.]: [s.n.], 1985.

KHALLOUFI, S.; GIASSON, J.; RATTI, C. Water activity of freeze dried mushrooms and berries. Canadian Agricultural Engineering, v. 42, n. 1, p. 7.1-7.13, 2000.

MADAMBA, P. S.; DRISCOLL, R. H.; BUCKLE, K. A. Thin-layer drying characteristics of garlic slices.Journal of Food Engineering, Essex, v. 29, n. 1, p. 75-97, 1996.

MOHSENIN, N.N. Physical properties of plant and animal materials. New York: Gordon and Breach Publishers, 1986. 841p.

MUDNIC,I.; MODUN,D.; BRIZIC, I.; VUKOVIC, J. Cardiovascular effects in vitro of aqueous extract of wild strawberry (Fragariavesca, L.). International Journal of Phytotherapy and Phytopharmacology, v.16, p.462-469, 2009.

STATSOFT, Inc. Statistica for Windows - computer program manual.Tulsa (OK): StatSoft, 1999.

RYAN, T. Estatística Moderna para Engenharia. $1^{\text {a }}$. ed.[S.1.]: Elsevier, 2009.

VAN DE BOER, J. H.The dynamical character of adsorption. Oxford: Clarendon Press. S.l. s.n. 1953.

VAN DEN BORG, C. Description of water activity of foods for engineering purposes by means of G.A.B. model sorption.Engineering and food, New York, v. 1, 1984. 